\title{
Results and Prospects for charm physics at LHCb
}

\author{
Philip John Hunt ${ }^{* \dagger}$ \\ University of Oxford, $U K$ \\ E-mail: p.hunt2@physics.ox.ac.uk
}

Precision measurements in charm physics offer a window into a unique sector of potential New Physics interactions. LHCb is an experiment designed for the high-statistics study of flavour physics. The conditions of the 2010-11 run of LHC are especially conducive to the collection of charm events, with a very large data-set already attained in 2010. First charm results will be shown using data collected at $\mathrm{LHCb}$ in pp collisions at $7 \mathrm{TeV}$ centre-of-mass energy, including measurements of the production cross-section and characterisation of the signal samples in key decay modes. The prospects for measurements of $\mathrm{CP}$ violation, mixing, and rare decays of charm mesons at LHCb will also be presented.

Kruger 2010: Workshop on Discovery Physics at the LHC

December 5 - 10, 2010

Kruger National Park, Mpumalanga, South Africa

* Speaker.

$\dagger$ On behalf of the LHCb collaboration 


\section{Introduction}

The LHCb detector is a single-arm forward spectrometer, with an approximate angular acceptance of $10<\theta_{x}<300 \mathrm{mrad}$ and $10<\theta_{y}<250 \mathrm{mrad}$ in the bending and non-bending planes respectively [1]. The experiment is designed to reconstruct individual $B$ events, which at LHC energies tend to be heavily boosted in the forward and backward directions. Crucial to the physics aims of LHCb are the detector subcomponents: the silicon strip Vertex Locator (VELO), whose unique design allows its insertion to within $8 \mathrm{~mm}$ from the beam during data-taking, providing excellent primary vertex (PV) resolution; a series of silicon pixel and drift chamber tracking stations situated before and after the $4 T \cdot m$ magnet; two Ring Imaging Cherenkov (RICH) detectors with three different radiators, giving exceptional discrimination between kaons and pions over a $2<|\vec{p}|<100 \mathrm{GeV} / c$ momentum range; five muon stations; an electron calorimeter with a preshower detector; and a hadronic calorimeter. The same features that make LHC ideal for $b$-physics are also important for charm physics.

LHCb's trigger system comprises a hardware first-level, designed to limit readout to a maximum of $1 \mathrm{MHz}$, and a two-stage software trigger. The trigger is primarily designed to select long-lived decays and so many triggered charm events originate from $B$ decays. However LHCb also has dedicated software trigger lines for selecting prompt charm decays, and the 2010 data includes a significant sample of these events. During 2010, the Large Hadron Collider (LHC) delivered an estimated luminosity of $42 \mathrm{pb}^{-1}$ to the LHCb detector with $p p$ collisions at $\sqrt{s}=7 \mathrm{TeV}$, of which approximately $90 \%$ was recorded. Results are presented in these proceedings with up to the full luminosity available at the end of 2010 running.

\section{Open charm production cross-section}

The first measurements of the cross-sections of the $D^{0}, D^{*+}, D_{s}^{+}$and $D^{+}$charmed mesons, as well as the total $c \bar{c}$ cross-section over the LHCb rapidity $(y)$ and transverse momentum $\left(p_{T}\right)$ range have been determined with the first $1.8 \mathrm{pb}^{-1}$ of $\sqrt{s}=7 \mathrm{TeV}$ collision data [2]. One of the key aspects of LHCb's measurement of the open charm cross-section as compared to the other LHC experiments is its capability of measuring the $D$ meson $p_{T}$ down to zero. The cross-section for each charm meson is determined in $y$ and $p_{T}$ bins and then integrated over the full phase-space. For the $D^{0}, D^{*+}$ and $D^{+}$measurements, eight $p_{T}$ bins of width $1 \mathrm{GeV} / c$ in the range $0<p_{T}<8 \mathrm{GeV} / c$, and five $y$ bins of width 0.5 in the range $2<y<4.5$ are used. Due to the low statistics for the $D_{s}^{+}$decay, the cross-section is measured in separate one-dimensional $y$ and $p_{T}$ bins. The current measurements include the following decays: $D^{0} \rightarrow K^{-} \pi^{+}, D^{*} \rightarrow\left(D^{0} \rightarrow K^{-} \pi^{+}\right) \pi^{+}, D^{+} \rightarrow K^{+} \pi^{+} \pi^{-}$and $D_{s}^{+} \rightarrow$ $\phi\left(K^{+} K^{-}\right) \pi^{+}$(throughout these proceedings, charge conjugates are implied).

The signal yield for a specific decay is determined by fitting the $D$ invariant mass distribution in each bin of $p_{T}$ and $y$. The level of contamination of the signal mass peak from secondary charm decays is determined from collision data by using a fit to the impact parameter (IP) distribution, with the prompt and secondary shapes determined from Monte Carlo (MC) generated signal decays. The selection and reconstruction efficiencies are determined from MC, with extensive cross-checks performed on the data. The efficiency of the particle identification (PID) cuts are determined from data using dedicated calibration data samples. The luminosity is determined using a combination 
of two techniques: measurement by the VELO of the beam profile using beam-gas events, which can be used to calculate the absolute luminosity [3], and dedicated runs in which a van der Meer scan [4] is performed. The uncertainty on the luminosity using these techniques is estimated to be $10 \%$ at present, and is dominated by the uncertainty of the measurement of the beam current. Another major contribution to the systematic error correlated between the various analyses are the tracking efficiencies, which have been determined from data to be $3 \%$ for high momentum tracks, and $4 \%$ for the soft pion track from $D^{*+} \rightarrow D^{0} \pi^{+}$. The measurements of the cross-sections in each of the bins in $p_{T}$ and $y$ are shown in Figure 1. The results show good agreement with theoretical predictions and the LHCb tuning of Pythia [5]. The results in various bins are combined to determine a value for the cross-section integrated over the measured $p_{T}$ and $y$ range for each charm meson. The probability for a charm quark to hadronise into a particular charmed meson as quoted by the PDG [6] from $e^{+} e^{-}$collisions around the $\Upsilon(4 S)$ resonance are then used to determine the $c \bar{c}$ cross-section in the same range. A combined average for the $c \bar{c}$ cross-section in the measured acceptance range is then calculated by a least-squares fit to a constant, accounting for correlations between the errors. The result for the $c \bar{c}$ cross-section is determined to be $1234 \pm 189 \mu \mathrm{b}$ over this pre-defined range. Finally, Pythia is used to extrapolate the result to the full $4 \pi$ of solid angle and full $p_{T}$ range, which results in a total $c \bar{c}$ cross-section of $6100 \pm 934 \mu \mathrm{b}$.

\section{Prospects for charm physics}

The key measurements in charm physics at LHCb during 2011 will $y_{C P}$ and $A_{\Gamma}$, which parameterise mixing and CP violation (CPV) respectively in the neutral $D$ sector. These measurements are outlined in Section 3.1, along with several longer-term analyses. In the charged charm sector, LHCb will also search for evidence of direct CPV in $D_{(s)}^{+} \rightarrow h^{+} h^{+} h^{-}$decays (where $h^{ \pm}$denotes a charged kaon or pion). These studies are outlined in Section 3.2. Other prospects for charm physics include the measurements of the $\psi(2 S)$ and $\chi_{c}$ charmonium cross-sections, and updated open charm cross-section results with increased statistics, including the first LHCb measurement of the $\Lambda_{c}^{+}$cross-section.

\subsection{Mixing and CPV in the neutral $D$ sector}

CPV in the neutral $D$ sector is parameterised by the relative magnitude $|q / p|$ and phase $\phi \equiv$ $\operatorname{Arg}(q / p)$ of the mass eigenstates. A discrepancy of the either the phase from zero, or the magnitude from unity implies CPV. Mixing between $D^{0}$ and $\bar{D}^{0}$ is parameterised by the variables $x$ and $y$. The first two charm mixing measurements made at $\mathrm{LHCb}$ will be of $y_{C P}$ and $A_{\Gamma}$, which are defined in terms of the mixing parameters by:

$$
\begin{gathered}
y_{C P}=\frac{\tau\left(D^{0} \rightarrow K^{-} \pi^{+}\right)}{\tau\left(D^{0} \rightarrow K^{+} K^{-}\right)}-1 \equiv(|q / p|+|p / q|) y \cos \phi-(|q / p|-|p / q|) x \sin \phi, \\
A_{\Gamma}=\frac{\tau\left(\bar{D}^{0} \rightarrow K^{+} K^{-}\right)-\tau\left(D^{0} \rightarrow K^{+} K^{-}\right)}{\tau\left(\bar{D}^{0} \rightarrow K^{+} K^{-}\right)+\tau\left(D^{0} \rightarrow K^{+} K^{-}\right)} \equiv(|q / p|-|p / q|) y \cos \phi-(|q / p|+|p / q|) x \sin \phi .
\end{gathered}
$$




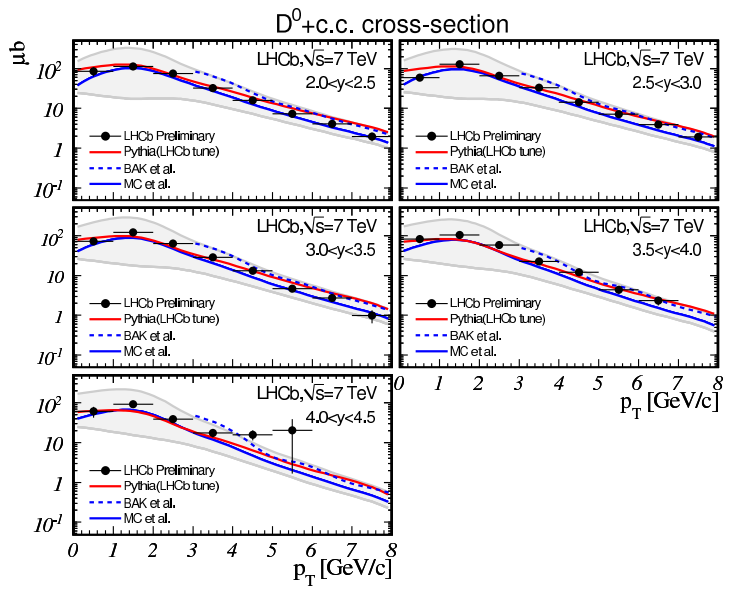

(a)

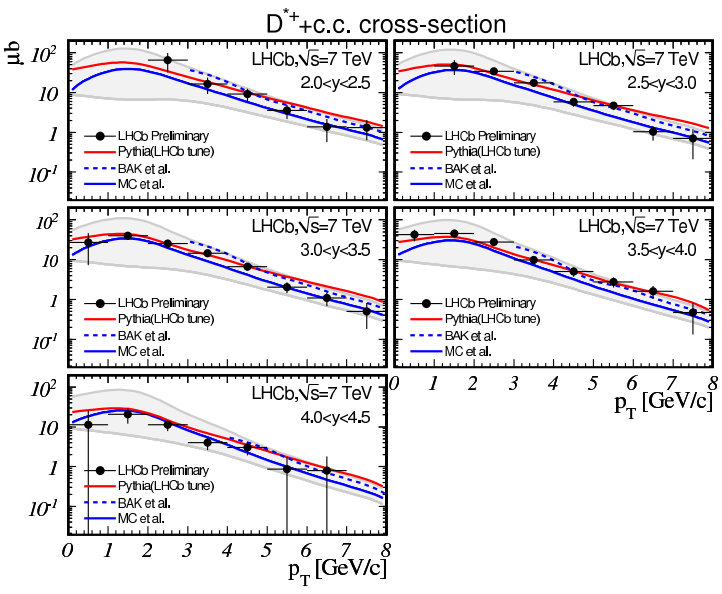

(b)

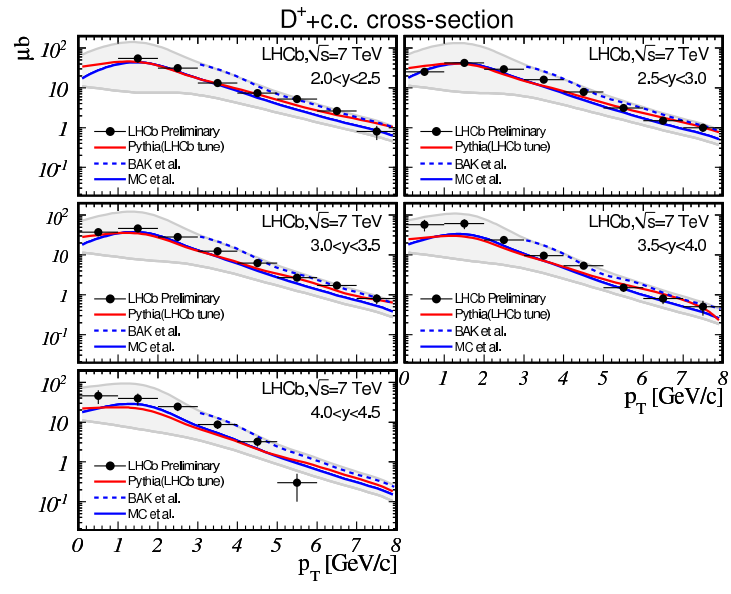

(c)

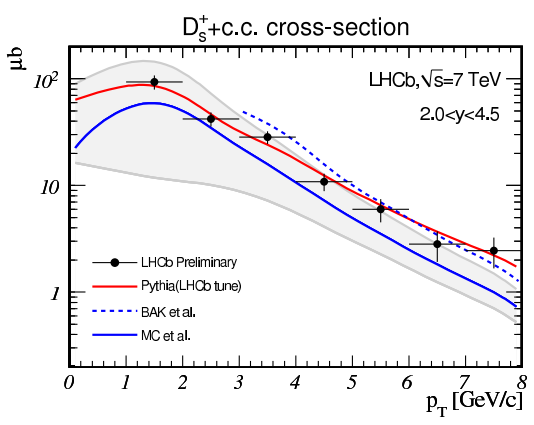

(d)

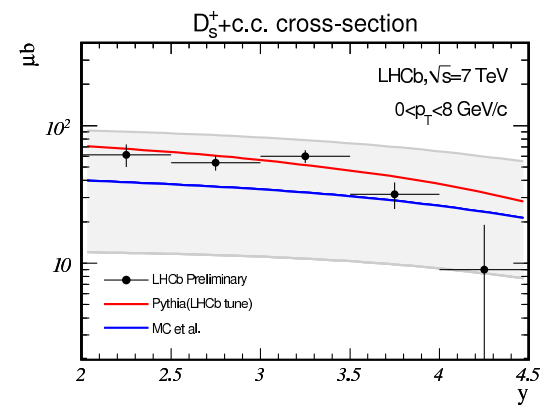

(e)

Figure 1: Measured cross-sections compared to theoretical predictions. The error bars show statistical and systematics errors which are uncorrelated between measurements added in quadrature. In addition, there are systematic errors that are partially or fully correlated between the different measurements. Figures (a), (b) and (c) show the measurements for the $D^{0}, D^{*}$ and $D^{+}$cross-sections as a function of $p_{T}$ for different ranges of $y$. The global systematic errors on these measurements are $12 \%, 14 \%$ and $14 \%$ respectively. Figures (d) and (e) show the measured $D_{s}^{+}$cross-sections for different ranges of $p_{T}$ integrated over $2<y<4.5$, and $y$ integrated over $0<p_{T}<8 \mathrm{GeV} / c$, respectively. The global systematic error on the $D_{s}^{+}$measurements is $16 \%$. The shaded areas are the theoretical uncertainties of the predictions by MC et al. 
The denominator of $y_{C P}$ can also be another CP eigenstate such as $D^{0} \rightarrow \pi^{-} \pi^{+}$. In the case of no CPV, $y_{C P}=y$, and $A_{\Gamma}=0 . y_{C P}$ can be measured from prompt $D^{0}$ decays, whilst $A_{\Gamma}$ requires that we know the flavour of the neutral $D$, which can be determined from the charge of the soft pion from the $D^{*} \rightarrow D^{0} \pi^{+}$decay, where the $D^{0}$ or $\bar{D}^{0}$ decays to the required final state. LHCb plans to measure $y_{C P}$ and $A_{\Gamma}$ using two independent techniques. The first involves measuring the ratio of yields in bins of proper time, utilising the fact that the limetime biases largely cancel. In the second method, unbinned lifetime measurements are performed, with the lifetime biases determined using a fully data-driven method [7]. The number of events from the first $25 \mathrm{pb}^{-1}$ of data analysed from the 2010 sample (requiring that the trigger fires on the signal decay), for prompt $D^{0} \rightarrow K^{-} \pi^{+}$and $D^{0} \rightarrow K^{+} K^{-}$decays are around 3 million and 0.4 million respectively. The corresponding yields for decays originating from a prompt $D^{*} \rightarrow D^{0} \pi^{+}$decay are approximately a factor 10 lower. This means that the 2010 data sample is large enough to yield competitive results with BELLE [8] and BABAR [9, 10] for the $y_{C P}$ measurement, and an analysis note is currently being prepared.

Another method for measuring mixing in the neutral $D^{0}$ sector utilises the proper-time distribution of the decay $D^{0} \rightarrow K^{+} \pi^{-}$. This decay can proceed either directly by the doubly Cabibbo suppressed (DCS) decay $D^{0} \rightarrow K^{+} \pi^{-}$, or via $D^{0}-\bar{D}^{0}$ mixing, followed by the Cabibbo favoured (CF) decay $\bar{D}^{0} \rightarrow K^{+} \pi^{-}$. The time evolution of this decay provides access to the mixing parameters $x^{\prime}$ and $y^{\prime}$, which are rotations of $x$ and $y$ with a CP conserving strong phase $\delta_{K \pi}$. Due to the low branching fraction of the DCS mode, $\approx 5$ times the integrated luminosity of the 2010 sample will be needed to be competitive with BABAR's measurement [11].

It is also possible to extract $x$ and $y$ directly (i.e. with no rotation by a strong phase) by performing a time-dependent Dalitz analysis of the decays $D^{0} \rightarrow K_{\mathrm{S}}^{0} h^{+} h^{-}(h=K, \pi)$. However, this is a rather a difficult and CPU intensive decay to trigger on, since the $K_{\mathrm{S}}^{0}$ is relatively long-lived, and often decays outside of the VELO (giving two so-called downstream tracks). To save time, the high-level trigger cuts tightly on the $h^{+} h^{-}$combination before searching for downstream tracks, however, this causes biases in the Dalitz space. Due to the low trigger efficiency, at least 10 times more data than the 2010 run will be needed to be comparable with previous analyses. However, because of the additional complication of systematic biases caused by the trigger strategy, this analysis will probably require the full 2011 statistics to make a competitive measurement.

Other interesting avenues for CPV in the neutral $D$ sector come from direct CPV resulting from new physics processes. Examples of direct CPV searches that are being persued come from the multi-body final state $D^{0} \rightarrow K^{+} K^{-} \pi^{+} \pi^{-}$, and the rare decay $D^{0} \rightarrow K^{+} K^{-} \mu^{+} \mu^{-}$. In this analysis, an observable that is odd under time-reversal is considered (such as the angle between the $K^{+} K^{-}$and $\pi^{+} \pi^{-}$or $\mu^{+} \mu^{-}$planes). A T-violating asymmetry is then constructed between the $D^{0}$ and $\bar{D}^{0}$. If this is non-zero, then this would indicate direct CPV. It is expected that a measurement of $D^{0} \rightarrow K^{+} K^{-} \pi^{+} \pi^{-}$will be possible with the 2011 data, providing that the trigger bandwidth in 2011 is consistent with 2010 running. $D^{0} \rightarrow K^{+} K^{-} \mu^{+} \mu^{-}$is too rare a decay to make this measurement with the expected luminosity from 2011 running. Instead, it is planned to measure the branching fraction. If the measured fraction is lower than the SM prediction, then this would suggest additional non-SM contributions in the decay amplitude, some of which could enhance CPV. 


\subsection{CPV in charged charm decays}

$\mathrm{LHCb}$ is also searching for direct CPV in charged charm meson decays, including the singly Cabibbo suppressed (SCS) $D^{+} \rightarrow K^{+} K^{-} \pi^{+}$and $D^{+} \rightarrow \pi^{+} \pi^{-} \pi^{+}$decay channels. These final states can proceed via several resonances, some of which can be CP violating. CPV would manifest itself in these modes by a charge asymmetry between the $D^{+}$and $D^{-}$in some of the resonant modes. Since the charge asymmetry can vary over the phase space, this analysis will use a model-dependent anisotropy method [12], where the charge asymmetry is evaluated using the $D^{+}$and $D^{-}$Dalitz plots on a bin-by-bin basis, with deviation of the significance, $\left(N_{D^{+}}-N_{D^{-}}\right) / \sqrt{N_{D^{+}}+N_{D^{-}}}$, from a Gaussian with $\mu=0$ and $\sigma=1$ indicating CPV. In order to distinguish between asymmetries due to $\mathrm{CPV}$, production, acceptance and trigger effects, the results are compared with Cabibbo favoured control channels with no predicted CPV, such as $D^{+} \rightarrow K^{-} \pi^{+} \pi^{+}$and $D_{s}^{+} \rightarrow \pi^{-} \pi^{+} \pi^{+}$. Preliminary studies of the primary search channel $D^{+} \rightarrow K^{+} K^{-} \pi^{+}$suggest that sensitivity to asymmetries up to the $10^{-3}$ level in the most favourable regions of Dalitz space will be possible with the full data-set collected at the end of the 2011 run, which would make this competitive with current measurements.

\section{Summary}

The preliminary measurements of the open charm cross-sections have been presented, and agree well with theoretical predictions and the LHCb tuning of Pythia. The key measurements of $y_{C P}$ and $A_{\Gamma}$ have also been discussed, in which LHCb will produce competitive results with 2010 data, and early 2011 data, respectively. Other analyses have also been outlined that will produce competitive results with the full or partial statistics of the 2011 run.

\section{References}

[1] LHCb Collaboration, The LHCb detector at the LHC, JINST 3 (2008) S08005.

[2] LHCb Collaboration, Prompt charm production in pp collisions at $\sqrt{s}=7 \mathrm{TeV}$, CERN-LHCb-CONF-2010-013 (2010) unpublished.

[3] H. Burkhardt and P. Grafstrom, Absolute Luminosity from Machine Parameters, CERN-LHC-PROJECT-REPORT-1019 (2007) unpublished.

[4] S. van der Meer, Calibration of the effective beam height in the ISR, CERN-ISR-PO/68-31 (1968) unpublished.

[5] T. Sjöstrand, S.Mrenna and P.Skands, PYTHIA 6.4 Physics and Manual, JHEP 05 (2006) 026.

[6] Particle Data Group, C. Amsler et al., Fragmentation Functions in $e^{+} e^{-}$Annihilation and Lepton-Nucleon DIS, in Review of Particle Physics, Phys. Lett. B667 (2008) 1.

[7] M. Gersabeck et al., A Monte Carlo simulation free method of measuring lifetimes using event-by-event acceptance functions at LHCb , LHCb-PUB-2009-022 (2009) unpublished.

[8] BELLE Collaboration, Evidence for $D^{0}-\bar{D}^{0}$ Mixing, Phys. Rev. Lett. 98 (2007) 211803.

[9] BABAR Collaboration, Measurement of $D^{0}-\bar{D}^{0}$ mixing using the ratio of lifetimes for the decays $D^{0} \rightarrow K^{-} \pi^{+}, K^{-} K^{+}$, and $\pi^{-} \pi^{+}$, Phys. Rev. D78 (2008) 011105. 
[10] BABAR Collaboration, Measurement of $D^{0}-\bar{D}^{0}$ mixing using the ratio of lifetimes for the decays $D^{0} \rightarrow K^{-} \pi^{+}$and $K^{+} K^{-}$, Phys. Rev. D80 (2009) 071103.

[11] BABAR Collaboration, Evidence for $D^{0}-\bar{D}^{0}$ Mixing, Phys. Rev. Lett. 98 (2007) 211802.

[12] I. Bediaga et al. On a CP anisotropy measurement in the Dalitz plot, Phys. Rev. D80 (2009) 096006. 